University of Wollongong

Research Online

Faculty of Engineering and Information

Faculty of Engineering and Information

Sciences - Papers: Part A

Sciences

$1-1-2016$

\title{
A data-driven predictive model for residential mobility in Australia - a generalised linear mixed model for repeated measured binary data
}

Mohammad-Reza Namazi-Rad

University of Wollongong, mrad@uow.edu.au

Payam Mokhtarian

University of Wollongong, payam@uow.edu.au

Nagesh Shukla

University of Wollongong, nshukla@uow.edu.au

Albert Munoz

University of Wollongong, amunoz@uow.edu.au

Follow this and additional works at: https://ro.uow.edu.au/eispapers

Part of the Engineering Commons, and the Science and Technology Studies Commons

Research Online is the open access institutional repository for the University of Wollongong. For further information contact the UOW Library: research-pubs@uow.edu.au 


\title{
A data-driven predictive model for residential mobility in Australia - a generalised linear mixed model for repeated measured binary data
}

\author{
Abstract \\ Household relocation modelling is an integral part of the Government planning process as residential \\ movements influence the demand for community facilities and services. This study will address the \\ problem of modelling residential relocation choice by estimating a logit-link class model. The proposed \\ model estimates the probability of an event which triggers household relocation. The attributes \\ considered in this study are: requirement for bedrooms, employment status, income status, household \\ characteristics, and tenure (i.e. duration living at the current location). Accurate prediction of household \\ relocations for population units should rely on real world observations. In this study, a longitudinal survey \\ data gathered in the Household, Income and Labour Dynamics in Australia (HILDA) program is used for \\ modelling purposes. The HILDA dataset includes socio-demographic information such general health \\ situation and well-being, lifestyle changes, residential mobility, income and welfare dynamics, and labour \\ market dynamics collected from the sampled individuals and households. The technique presented in this \\ paper links possible changes in households' socio-demographic characteristics to the probability of \\ residential relocation by developing a mixed effects discrete-choice logit model (MEDCLM) for \\ longitudinal binary data using the HILDA dataset. The proposed model captures the effect of repeated \\ measurements together with the area-specific random effects.
}

\section{Keywords}

residential, model, predictive, binary, measured, repeated, driven, mixed, data, linear, generalised, australia, mobility

Disciplines

Engineering | Science and Technology Studies

\section{Publication Details}

Namazi-Rad, M., Mokhtarian, P., Shukla, N. \& Munoz, A. (2016). A data-driven predictive model for residential mobility in Australia - a generalised linear mixed model for repeated measured binary data. Journal of Choice Modelling, Online First 1-12. 


\title{
A Data-Driven Predictive Model for Residential Mobility in Australia - A Generalised Linear Mixed Model for Repeated Measured Binary Data
}

\author{
Mohammad-Reza Namazi-Rad ${ }^{\mathrm{a}, *}$, Payam Mokhtarian $^{\mathrm{b}}$, Nagesh Shukla $^{\mathrm{c}}$, \\ Albert Munoz ${ }^{\mathrm{d}}$ \\ ${ }^{a}$ National Institute for Applied Statistics Research Australia, University of Wollongong, \\ NSW 2522, Australia \\ ${ }^{b}$ Singtel Optus Pty Ltd, NSW 2113, Australia \\ ${ }^{c}$ SMART Infrastructure Facility, University of Wollongong, NSW 2522, Australia \\ ${ }^{d}$ School of Management, Operations \& Marketing, University of Wollongong, NSW, 2522, \\ Australia
}

\begin{abstract}
Household relocation modelling is an integral part of the Government planning process as residential movements influence the demand for community facilities and services. This study will address the problem of modelling residential relocation choice by estimating a logit-link class model. The proposed model estimates the probability of an event which triggers household relocation. The attributes considered in this study are: requirement for bedrooms, employment status, income status, household characteristics, and tenure (i.e. duration living at the current location). Accurate prediction of household relocations for population units should rely on real world observations. In this study, a longitudinal survey data gathered in the Household, Income and Labour Dynamics in Australia (HILDA) program is used for modelling purposes. The HILDA dataset includes socio-demographic information such general health situation and wellbeing, lifestyle changes, residential mobility, income and welfare dynamics, and labour market dynamics collected from the sampled individuals and households. The technique presented in this paper links possible changes in households' socio-demographic characteristics to the probability of residential relocation by developing a mixed effects discrete-choice logit model (MEDCLM) for longitudinal binary data using the HILDA dataset. The proposed model captures the effect of repeated measurements together with the area-specific random effects.
\end{abstract}

Keywords: Behavioural Model; HILDA Data; Household Relocation Modelling; Mixed Effects Discrete-Choice Logit Model (MEDCLM); Repeated Measurements.

\footnotetext{
is

*Corresponding author:Mohammad-Reza Namazi-Rad Email: mrad@uow.edu.au
} 


\section{Introduction}

Household residential relocation decisions are influence community make-up and population levels in different geographical areas across countries. The risk of inefficient public policies and poor Government service provisions can be reduced by accurately modelling the location choices for large proportion of a population. At the same time, sporadic location choices decreases the reliability of traditional equilibrium-based approaches to modelling population movements. Changes in household configurations, individual attributes, and community structures strongly affect the demand for public services in regional and urban areas. Thus, planners are under increasing pressure to develop robust policies that provide adequate levels of the right services.

Traditionally, residential relocation has been modelled using aggregate forecasting techniques. However, many assumptions supporting these models can be ineffective when applied to specific socio-demographic population segments, increasing the need to adopt more sophisticated, robust planning tools based on peer-reviewed research. There are many relevant research studies in areas such as social psychology (Oishi and Schimmack (2010)), demography (South and Crowder (1997)), epidemiology (Jelleyman and Spencer (2008)) and other social and behavioural sciences (Sergeant et al. (2008)). Models of relocation typically require region-specific attributes as cultural and local geography factors can play a significant role in relocation decisions ( $\mathrm{Hu}$ et al. (2008)). Examples include tenure at the current residential location and the perceived net benefit of relocation.

This study presents a data-driven mixed effects model with repeated measurements to to determine if and when a household (in Australia) initiates the relocation process. It is assumed that all households can make relocation choices whenever relevant changes in socio-demographic and area-level factors sufficiently necessitate the initiation of this process. For example, a household may be in a situation whereby a change in job location may require a longer com- 
mute, triggering the possibility of relocation process to reduce commute time. The increased commute time will translate to an increase in their willingness to relocate. However, if changes to the job situation are not significant enough, the household may not initiate the relocation process. Similar models are presented in studies on land-use and residential mobility (Kan (1999), Vega and ReynoldsFeighan (2009), Pagliara and Wilson (2010), Ibeas et al. (2013), Jun (2013)). The novelty of the modelling technique presented in the current study is in the inclusion of area-specific mixed effects together with the effect of repeated measurements.

In this paper, a certain class of behavioural models is discussed for modelling the trigger for residential movements using the Household, Income and Labour Dynamics in Australia (HILDA) longitudinal survey data while considering the effect of area of living and repeated measurements. The dataset used for the model presented in this paper comes from the Australian Government Department of Families, Housing, Community Services and Indigenous Affairs (FaHCSIA). FaHCSIA initiated the HILDA program to gather reliable longitudinal data on family and household dynamics. In this survey socio-demographic information (e.g. general health situation and well-being, long term lifestyle changes, residential mobility, income and welfare dynamics, and labour market dynamics) is collected from selected sampled individuals and households living in different parts of Australia. The Melbourne Institute of Applied Economic and Social Research currently manages the HILDA project and the data repository (Watson and Wooden (2014)).

\section{Self-reported Area-specific Residential Mobility in Australia}

Arguably, economic models have been the only conceptually consistent and analytically tractable framework to model residential relocation dynamics. In the urban economics context, a willingness-to-pay driven framework relies on five axioms that to provide its consistency: $i$ ) prices adjust to achieve local equi- 
librium, ii) self-reinforcing effects generate extreme outcomes, iii) externalities cause inefficiency, $i v$ ) production is subject to economies of scale and $v$ ) competition generates zero economic profit (OSullivan (2009)). This approach has been criticised by its reductionism, supported by arguments that residential relocation choices encompass factors like social bonding or 'sense of place' that hardly fit into a single currency framework. Moreover, assumptions of perfect competition, economies of scale, and equilibrium markets tend to reduce the validity of conclusions inferred from such models.

Louviere and Meyer (2008) proposed to forge a better alliance between economic theories and behavioural research in order to improve representation of informal choices within a discrete choice-modelling paradigm. A common methodology used in discrete choice modelling is logit class models, whereby a number of alternatives are evaluated by the probability of each alternative being chosen by an individual autonomous entity. Here, the main objective is to use a datadriven statistical technique to model the trigger for residential movements in different parts of Australia (where HILDA survey data is available) while considering the effect of time and other important factors in residential mobility relevant to the context of this study. The model estimates the probability of a household choosing to relocate and implicitly initiating the relocation process. The attributes considered in this study are: requirement for bedrooms, employment status, income status, household characteristics, and tenure (i.e. duration living at the current location). HILDA survey data is used for modelling the location choice trigger in Australia.

Cursory data analysis indicates that around $17 \%$ of the total households and $13 \%$ of couple families in the HILDA survey relocate each year. Figure 1 demonstrates the proportion of residential relocations within a year in Australian major metropolitan areas from 2001 to 2011. There are noticeable fluctuations in area-specific movements. For example, the proportion of movements in Darwin peaks in 2004-2005, perhaps due to the major development project initiatives in Darwin at the time. Most notably, the redevelopment of the Wharf Precinct 
and associated new housing developments including Outrigger Pandanas and Evolution on Gardiner (NorthernTerritoryGovernment (2007)). For Tasmania, the influence of permanent migrants during 2004-2005 represented a significant increase of 141 residents from 2003-2004, and 278 more people than in 2002-2003 (Minnucc (2008)).

The decrease in Canberra residents between 2003 and 2004 may be due to bushfire events. In January 2003 severe weather triggered catastrophic bushfire that destroyed around 500 homes. In reaction to the disaster, the Canberra Spatial Plan for the city's future development was released in 2004. Plans included a new Canberra district to be situated west of Lake Burley Griffin to encourage commercial and residential growth. Although the number of residential movements at the Australia's major metropolitan areas has fluctuated from 2002 to 2011 , on average the proportion of total households moved was between $17 \%$ and $23 \%$ in 2011 except Darwin for which this proportion was approximately closer to $38 \%$. Other objective and subjective factors not captured in this study would undoubtedly influence movements in different parts of Australia. The cursory analysis presented on Figure 1 presents some of the qualitative assessments of the model of trigger for residential movements will attempt to formalise. 
Figure 1: Self-reported area-specific movements at the Australian big cities within a year (2001-2011) recorded by HILDA

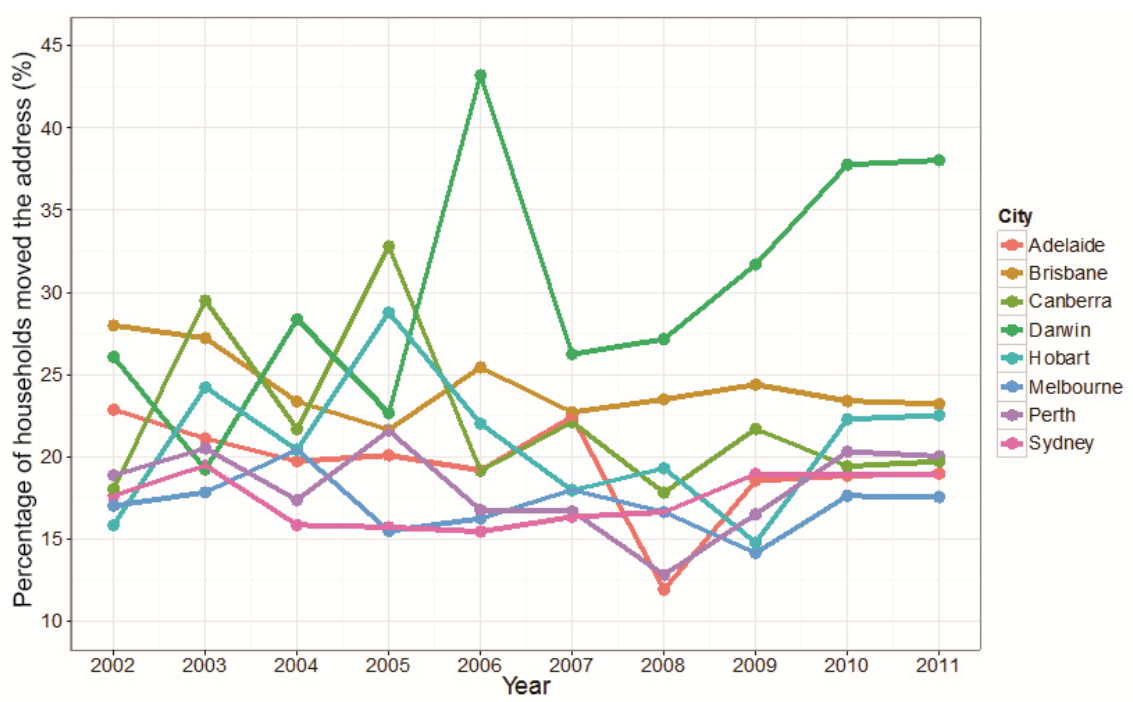

A limitation of the Figure 1 data analysis is that factors not captured in this study would also influence mass household relocations to different parts of Australia. However, the use of more accurate, and perhaps more sophisticated models, to further explore into the link between catastrophic events or governmentdriven redevelopment initiatives provides an exciting future research direction for this research.

\section{Behavioural Models}

The goal of behavioural models and in particular discrete choice models is to predict how different factors and the behavioural process collectively determine, or cause, the individual's (i.e., person, firm, or a decision maker unit) choice and to derive the probability of any particular outcome (Anderson et al. (2013)). Using such models in cases where we are interested in determining whether or not an individual takes a particular action, such as residential relocation, the choice probability can be calculated exactly from a closed-form formulation. 
To describe the complexity of behavioural processes by which an individual makes a choice, or a series of choices over time (among a well-defined set of options), discrete choice models are derived. These models typically adopt the assumption that human beings will exhibit utility-maximising behaviour. This concept in terms of psychological stimuli was originally developed by Thurstone (1927), leading to a probit model of whether respondents differentiate the level of stimulus. Further, Marschak (1960) interpreted the stimuli as utility and provided a derivation from utility maximisation.

Discrete choice models have received an increasing attention in the applications of residential mobility and transportation. For the purpose of modelling residential mobility, the class of discrete choice modelling based on logit models and its variations are used predominantly in the literature for modelling the trigger for residential mobility or residential location choices (e.g. Bhat and Guo (2004), Lee and Waddell (2010)). In this section, a mixed model of two-option choice is presented when using longitudinal survey data.

\subsection{Linear Mixed-Effects Models}

Survey sampling methods were developed in the first half of the last century in order to provide statistical techniques for conducting appropriate sample designs and describing the process of selecting sample individuals from the target population and producing estimates (Chambers and Clark (2012)). Usually, sample information is not sufficient for direct estimation purposes in small areas due to small sample sizes. Therefore, resulting in unrealiable area-specific direct estimators. Indirect techniques are used in such cases using linking models with other data sources in order to achieve required estimators with acceptable quality (Namazi-Rad and Steel (2015)).

Here, we consider a population of size $N$ divided into $K$ small areas with $N_{k}$ agents (e.g. individuals, households) in the $k$ th small area $\left(N=\sum_{k=1}^{K} N_{k}\right)$ from which a sample of size $n_{k}$ participated in the survey. The total sample size out of the whole population is therefore equal to $n=\sum_{k=1}^{K} n_{k}$. For the 
purpose of modelling the survey data in the current study, an indicator function is defined that takes only two values; i.e. 0 or 1 . This function takes the value of 1 , indicating that the $i$ th unit (or agent) at the $k$ th area chooses the choice 'a' (among a well-defined set of options,) which leads to the outcome $y_{i k}^{(a)}$; i.e. $I\left(y_{i k}^{(a)}\right)=1: i \in\left\{1, \ldots, n_{k}\right\} \& k \in\{1, \ldots, K\}$. Alternatively, this indicator function takes the value of 0 , indicating that the agent chooses an another outcome; i.e. $I\left(y_{i k}^{(a)}\right)=0$.

For $i$ th individual at $k$ th area, the unit-level random utility model (RUM) is presented in the literature (Marschak (1960), Train (2009), Claassen et al. (2013)) to relate the probability of choosing the choice ' $a$ ' denoted by $\pi_{i k}^{(a)}$ (with the logit-link function $\left.\eta_{i k}^{(a)}=\operatorname{logit}\left(\pi_{i k}^{(a)}\right)\right)$ with $q$ auxiliary variables at the unit level (i.e., individual or agent) as follows

When utilizing binary data (as discussed by Benedetti et al. (2014)), the unitlevel random utility model (RUM) is presented in the literature (e.g. Train (2009), Claassen et al. (2013)) to relate the probability of choosing the outcome $y_{i k}^{(a)}$ denoted by $\operatorname{Pr}\left(I\left(y_{i k}^{(a)}\right)=1 \mid \mathbf{x}_{i k}\right)=\pi_{i k}^{(a)}$ with the vector of $P$ auxiliary variables at the unit level denoted by $\mathbf{x}_{i k}^{\top}=\left[\begin{array}{llll}1 & x_{i k 1} & x_{i k 2} \ldots x_{i k P}\end{array}\right]$. The binary logit-mixed model of a two-option choice behaviour is demonstrated as follows:

$$
\eta_{i k}^{(a)}=\mathbf{x}_{i k}^{\top} \boldsymbol{\beta}+\epsilon_{i k}
$$

with the link-function $\eta_{i k}=\operatorname{logit}\left(\pi_{i k}\right)$. In equation (1), $\boldsymbol{\beta}$ is the vector of model coefficients and $\epsilon_{i k}=u_{k}+e_{i k}$, where $e_{i k}$ denotes the model error associated with the $i$ th unit within $k$ th area with zero-mean and variance $\sigma_{e}^{2}$, while $u_{k}$ is the $k$ th area-specific random effect with zero-mean and variance $\sigma_{u}^{2}$.

The aim of building the model presented in the form of equation (1) is for this model to be best fitted on the sample data for which the model parameters should be estimated accurately. Then, the variable of interest in the model is predicted as $\hat{\eta}_{i k}^{(a)}=\mathbf{x}_{i k}^{\top} \hat{\boldsymbol{\beta}}+\hat{u}_{k}$ and

$$
\hat{\pi}_{i k}^{(a)}=\operatorname{logit}^{-1}\left(\hat{\eta}_{i k}^{(a)}\right)=\frac{1}{1+e^{-\hat{\eta}_{i k}^{(a)}}} .
$$


Here, $\eta_{i k}$ is assumed to be distributed logistically with the density function $f\left(\eta_{i k}\right)=e^{-\eta_{i k}}\left(1+e^{-\eta_{i k}}\right)^{-2}$. Unlike the identity link-function, the logit-link function will always yield estimated means in the range of zero to one while the effect of one unit change in the linear predictor is not constant.

\subsection{Logit-Mixed Model for Repeated Measurements}

Longitudinal surveys have been used in research studies that involve repeated observations of the same variables over long periods of time. Longitudinal studies in sociology generally aim to study life events throughout lifetimes or generations by tracking population units (e.g. individuals, households) at multiple times (Wall and Williams (1970)). Many of the variables of interest in longitudinal research studies are nominal variables with two or more categories (e.g., employment status, occupation, political preference, or self-reported health status) as discussed in Haynes et al. (2005). In this method, the differences observed in those population units are less likely to be the result of cultural differences across generations (Hedeker and Gibbons (2006)).

Generalised linear models (GLMs) and generalised linear mixed models (GLMMs) have attracted considerable attention over the years to model longitudinal social, psychological, and medical data. This is while the element of time is mostly considered to refer to as the random effects when using GLMMs for modelling longitudinal data (?, Gad and Kholy (2012), Chiou et al. (2012)). Alternatively, the repeated measurements in a longitudinal study can be modelled using a GLMM where the correlation structure within each time sequence of measurements can be included using parameters for measurement error, variation between experimental units, and serial correlation within units (?, Diggle et al. (2008)). In this study, the unit-level mixed effects discrete-choice logit model (MEDCLM) that incorporates the repeated measurements discussed above is

$$
\eta_{i k}=\mathbf{x}_{i k}^{\top} \boldsymbol{\beta}+u_{k}+\Delta_{i k}(t)+e_{i k}^{(t)},
$$


where $\Delta_{i k}(t)$ denotes the repeated measurement effect for the $i$ th unit within $k$ th area from which information is recorded in the data more than once.

The repeated measurement effect represents the emergent inherent correlated errors within each erea over the time. This effect follows a Gaussian process with a correlated variance-covariance matrix. Given response data which is assessed over time, the extra repeated measurement term in the GLMM results in higher accuracy as it can capture more variability when a model is fitted Diggle et al. (2008). The empirical effect of the repeated measurement is presented in Section 4.

The main objective in this paper is to use the MEDCLM presented in the form of equation (3) for modelling the trigger for residential movements. This is done by fitting the equation (3) on available HILDA survey data, based on which model coefficients and model errors are to be estimated and model random effects are to be predicted. This will happen through an iterative process which includes the estimation of variance-covariance elements. In the statistical literature, the random effects and model errors are mostly considered as to be independent and identically distributed (IID) with means equal to zero. In many studies (e.g. Diggle et al. (2008)), multivariate normal distribution is considered for model errors and random effects for the purpose of model fitting as a fundamental assumption. Although the model errors and random effects are following this distribution in our paper, this was not considered as the fundamental assumption in the numerical approach employed for estimating model parameters.

\subsection{Model Parameter Estimation and Measurement of Performance}

According to Zeger and Liang (1986), when using GLLMs, it is not always possible to derive the log-likelihood of the data and approximations are needed to evaluate the log-likelihood function. However, regression coefficients in a GLMM can be estimated by solving the generalised estimating equations (GEEs) as fol- 
lows

$$
\sum_{k} \sum_{i} \frac{\partial \eta_{i k}}{\partial \mu_{\beta_{k}}} \mathbf{V}_{k}^{-}\left(\eta_{i k}-\mu_{\eta_{k}}\right)=0
$$

where $\mu_{\eta_{k}}=E\left(\eta_{k} \mid \mathbf{x}_{k}\right)$ is the model-specific area-level means and generalised inverse of the variance-covariance matrix as in: (Demidenko (2013))

$$
\mathbf{V}_{k}=\underbrace{\mathbf{W}_{\Delta_{k}(t)}^{\prime} \otimes \mathbf{G}_{k} \otimes \mathbf{W}_{\Delta_{k}(t)}}_{\mathcal{W}_{k}}+\mathbf{R}_{k}
$$

Here, $\mathbf{R}_{k}$ denotes the individual error variance within area $k$ and $\mathcal{W}_{k}$ denotes the correlated variance-covariance structure of area-specific effects with repeated measurements over the time where $\otimes$ is the Kronecker product. Detailed discussions are presented in the Appendix. When $\mathbf{V}_{k}$ is not identifiable, the variance of the response is assumed to depend on the mean for all families and have the scale parameter as a multiplier. The Newton-Raphson method is then used to calculate the quasi-likelihood estimators of model parameters, iteratively. The original quasi-likelihood (QL) function was introduced by Wedderburn (1974) to describe a function with similar properties to the log-likelihood function while the QL function is not the log-likelihood corresponding to any actual probability distribution. This method starts with an initial value for the vector of model coefficients $\boldsymbol{\beta}$ denoted by $\hat{\boldsymbol{\beta}}^{(0)}$. Based on Mccullagh 1983) and Ahmed and Fallahpour (2012), the $r$ th step of this iterative method results in an adjustment to $\hat{\boldsymbol{\beta}}^{(r-1)}$ using the following equation.

$$
\hat{\boldsymbol{\beta}}^{(r)}-\hat{\boldsymbol{\beta}}^{(r-1)}=\left(\mathbf{D}^{\top} \mathbf{V}^{-} \mathbf{D}\right)^{-} \mathbf{D}^{\top} \mathbf{V}^{-}\left(\eta-\hat{\mu}_{\eta}^{(r-1)}\right) .
$$

The iteration continues until convergence. Here,

$$
\mathbf{D}=\frac{\partial \mu_{\eta}}{\partial \boldsymbol{\beta}}
$$

and $\mathbf{V}$ is a block diagonal matrix with $\mathbf{V}_{k}$ diagonal-matrix elements.

To assess the performance of the modelling outcomes, accuracy of random effect predictions are calculated. Accuracy and reliability of predictions given a fitted model depend on the total amount of variability among observations captured by 
the model parameter estimators and random effect predictors. Predicting model random effects and estimating variance components are the most effective way of expressing the variability among observations captured by the fitted model. The accuracy of predictions is generally measured by the correlation between true and predicted random effects denoted by ' $r$ '.

However, the accuracy of random effect predictions is usually expressed in terms of reliability, with the squared correlation between true and predicted random effects, dented by $r^{2}$. The calculations needed for $r^{2}$ require the prediction error variance $(\mathrm{PEV})$ of the random effects (Henderson (1975)). Given the working model presented in (3), the area-specific effects represent the variability among observations whereas temporal effects can only represent the variability trend over time (measurements). Therefore, model accuracy in terms of prediction needs to be evaluated based on the accuracy of area-specific effect predictions. Hence, PEV estimation for area $k$ is calculated as a variability measurement for evaluating the accuracy of the predicted effect for this area as follows

$$
P E V_{k}=\operatorname{Var}\left(u_{k}-\hat{u}_{k}\right)=c_{k} \hat{\sigma}_{e}^{2},
$$

where $c_{k}$ is

$$
c_{k}=\frac{\left(n-\sum_{k=1}^{n_{k}} x_{i k}^{2}\right) \hat{\sigma}_{u}^{2}+\hat{\sigma}_{e}^{2}}{\hat{\sigma}_{u}^{2} \hat{\sigma}_{e}^{2}} .
$$

The PEV estimation could be regarded as the fraction of area effects variance not accounted for by the prediction. Therefore, using the variance components, it could be expressed as:

$$
P E V_{k}=\left(1-r_{k}^{2}\right) \hat{\sigma}_{u}^{2},
$$

where $r_{k}^{2}$ is the squared correlation between the true and predicted $k$ th area effect. Hence, the average model accuracy (in terms of the area-specific variability) is as follows

$$
r^{2}=\frac{1}{K} \sum_{k=1}^{K}\left(1-c_{k} \frac{\hat{\sigma}_{e}^{2}}{\hat{\sigma}_{u}^{2}}\right)
$$

The accuracy measures for area-specific model predictions as in 10 are used to compare different models in terms of the relative performance. Additionally, this 
measure can be used to express the accuracy in prediction validation. In fact, this measurement represents the proportion of variability among observations captured by a fitted model. Generally speaking, the larger the model accuracy value, the stronger the evidence for model accuracy and reliability in terms of prediction.

\section{Modelling the Trigger for Residential Re-Location in Australia}

The HILDA data allows us to observe the reasons for household movements in Australia. The aggregated categories and the specific reasons for moving the residential address are listed in Table 1. The socio-economic indexes for areas (SEIFA) are defined by Australian Bureau of Statistics (ABS) in 2004 and 2008 (ABS (2004); ABS (2008)) and serve to classify the reasons for residential relocations in Australia. According to the HILDA data, couples living in all SEIFA areas are most likely to move to a more expensive house. The most important reason to move for singles living in middle or high SEIFA areas is lifestyle, while family issues are the most important drivers of residential mobility for

households in low SEIFA areas (Black et al. (2009)). Here, we consider the categories listed in Table 1 in modelling the trigger for a residential move. 
Table 1: Definition of aggregate categories of reasons for moving (HILDA)

\begin{tabular}{|c|c|}
\hline Aggregate categories $(\gamma)$ & Includes the listed reasons \\
\hline Lifestyle $\left(\gamma_{1}\right)$ & $\begin{array}{l}\text { To get place of my own } \\
\text { To live in better neighbourhood } \\
\text { Amenities } \\
\text { Lifestyle } \\
\text { Neighbourhood reasons }\end{array}$ \\
\hline Family $\left(\gamma_{2}\right)$ & $\begin{array}{l}\text { To get married } \\
\text { Closer to friends and family } \\
\text { Marital breakdown } \\
\text { Whole family moved } \\
\text { Personal / Family }\end{array}$ \\
\hline Enforced $\left(\gamma_{3}\right)$ & $\begin{array}{l}\text { Property no longer available } \\
\text { Evicted } \\
\text { Temporary relocation } \\
\text { Government housing (no choice) }\end{array}$ \\
\hline Job related $\left(\gamma_{4}\right)$ & $\begin{array}{l}\text { To start job with new employer } \\
\text { Nearer place of work } \\
\text { Work transfer } \\
\text { Start own business } \\
\text { Relocate own business } \\
\text { Work reasons }\end{array}$ \\
\hline Cheaper house $\left(\gamma_{5}\right)$ & To get smaller cheaper place \\
\hline More expensive house $\left(\gamma_{6}\right)$ & To get larger, better place \\
\hline Look for work $\left(\gamma_{7}\right)$ & Look for work \\
\hline Health $\left(\gamma_{8}\right)$ & Health \\
\hline Other $\left(\gamma_{9}\right)$ & $\begin{array}{l}\text { Close to place of study } \\
\text { Moved to Australia } \\
\text { Returned from overseas } \\
\text { Other }\end{array}$ \\
\hline
\end{tabular}

Figure 2 presents the proportion of HILDA survey participants who have moved their residential address from 2010 to 2011 in the Statistical Local Areas (SLAs), for which the HILDA data is available. 
Figure 2: Map of SLA-specific probability of residential mobility within a year (2010-2011) recorded by HILDA

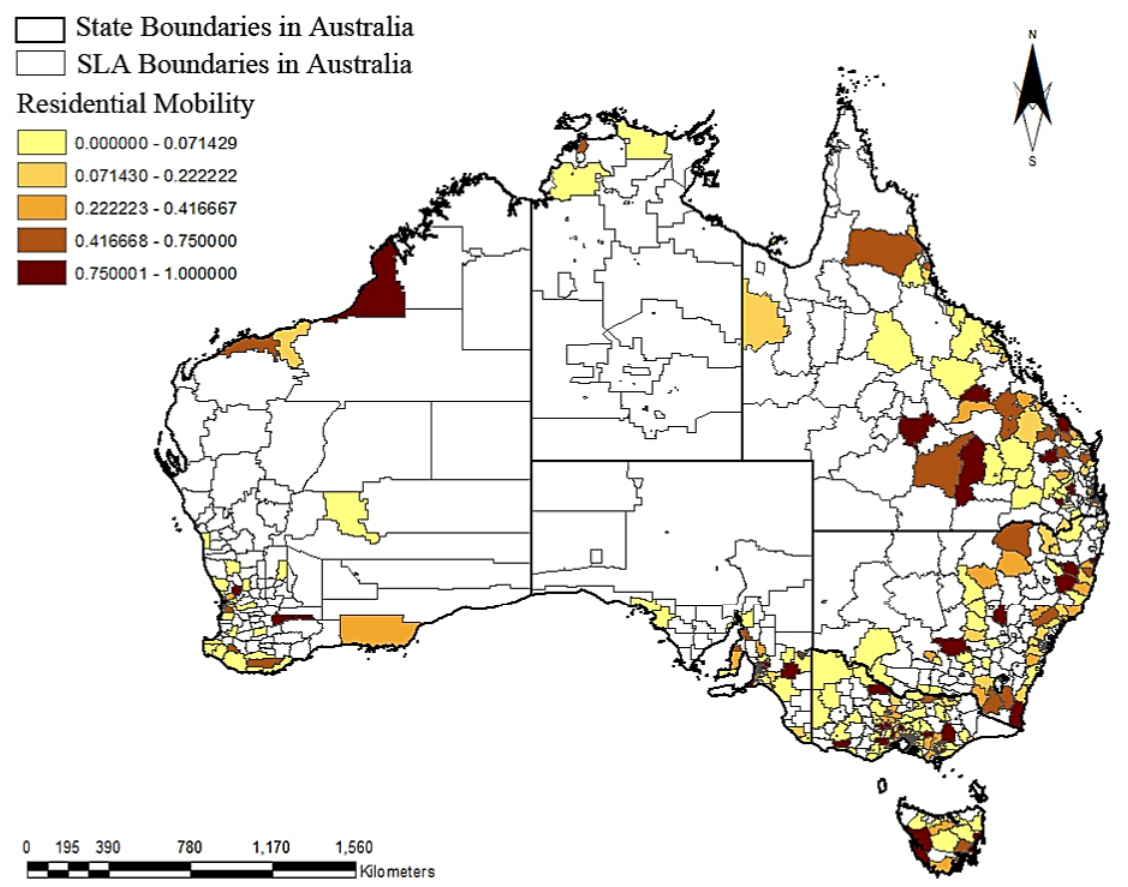

In total, more than 60000 households living in the SLAs presented in Figure 2 (out of 1034 SLAs in Australia) repeatedly participated in the HILDA survey between 2001 to 2011. The MEDCLM used in this paper to estimate the probability of an $i$ th household located at $k$ th SLA choosing to relocate and initiating the relocation process. This probability is denoted by $\pi_{i k}^{(R)}$. Attributes contributing to the relocation decision are: changes to number of bedrooms required, income level, household configuration, and tenure. The logit model of location choice trigger is as follows:

$$
\begin{aligned}
\eta_{i k}^{(R)}=\beta_{0}+\beta_{1} \mathcal{L}[ & \left.D_{i k}^{(B R)}-S_{i k}^{(B R)}\right]+\beta_{2} W_{i k}+\beta_{3} Q_{i k} \\
& +\mathbf{H H T}_{i k}^{T} \boldsymbol{\alpha}_{1}+\gamma_{i k} \boldsymbol{\alpha}_{2}+\Delta_{i k}(t)+u_{k}+e_{i k}^{(t)},
\end{aligned}
$$

where $\eta_{i k}^{(R)}$ is the linear predictor and is modelled by the inverse logistic link 
function

$$
\eta_{i k}^{(R)}=\log \left[\frac{\pi_{i k}^{(R)}}{1-\pi_{i k}^{(R)}}\right] \Rightarrow \pi_{i k}^{(R)}=\frac{1}{1+e^{-\eta_{i k}^{(R)}}},
$$

and

$$
\mathcal{L}(z)= \begin{cases}\log (z) & \text { if } z \geq 0 \\ -\log (z) & \text { if } z<0\end{cases}
$$

Here, $W_{i k}$ denotes the household annual net income of the $i$ th household at $k$ th SLA and $Q_{i k}$ is tenure for this household. Tenure for each household in this study is referred to as number of years lived in the current address.

Number of bedrooms in the residence at the current address is denoted by $S_{i k}^{(B R)}$, while $D_{i k}^{(B R)}$ denotes the number of bedrooms expected to be required for the household, depending on the size and composition. For $i$ th household at $k$ th, $D_{i k}^{(B R)}$ denotes the average number of the bedrooms in similar households in the same SLA. Notably, the difference between the current number of bedrooms for a household and the expected number of bedrooms calculated for this household can be either positive or negative. This is while most Australian participants in the HILDA survey have moved to residences with a larger number of bedrooms. While the distribution of number of bedroom reported in HILDA follows a Poisson distribution (see Figure 3), the logarithm function is used for this explanatory variable in the model 12 to decrease the effect of large values with low frequencies.
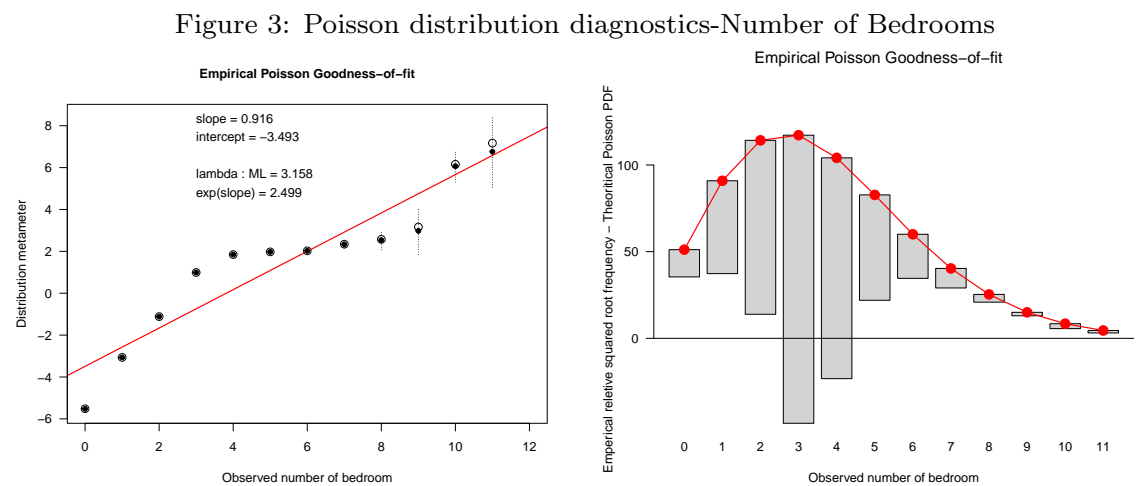
The aggregate category of reason for moving recorded for $i$ th household in the

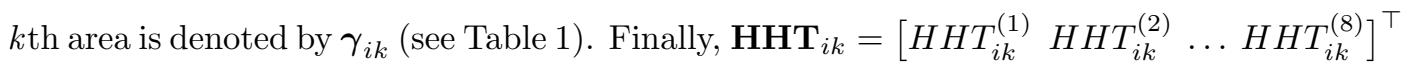
contains indicator variables corresponding to household type categories. This way, each sampled individual is allocated to a category of household type out of the categories presented in Table 2 .

Table 2: Household type categories

\begin{tabular}{||c|c||c|c||}
\hline \hline$H H T_{i k}^{(1)}$ & Lone person & $H H T_{i k}^{(5)}$ & $\begin{array}{c}\text { Lone parent with Children aged } \\
\text { less than 15-with and without Others }\end{array}$ \\
\hline$H H T_{i k}^{(2)}$ & $\begin{array}{c}\text { Couple family without Children-with } \\
\text { and without Others }\end{array}$ & $H H T_{i k}^{(6)}$ & $\begin{array}{c}\text { Lone parent with dependent or/and } \\
\text { non-dependent Children aged over 15- } \\
\text { with and without Others }\end{array}$ \\
\hline$H H T_{i k}^{(3)}$ & $\begin{array}{c}\text { Couple family with Children aged less } \\
\text { Group household \& Multi-family }\end{array}$ & $H H T_{i k}^{(7)}$ & $\begin{array}{c}\text { Group household \& Multi-family } \\
\text { household }\end{array}$ \\
\hline$H H T_{i k}^{(4)}$ & $\begin{array}{c}\text { Couple family with dependent or/and } \\
\text { non-dependent Children aged over } \\
\text { 15-with and without Others }\end{array}$ & $H H T_{i k}^{(6)}$ & Other related family without children \\
\hline \hline
\end{tabular}

The model parameters in (12) are: $u_{k}$ is the random effect for the $k$ th SLA; $e_{i k}$ is the random error for the $i$ th household within the $k$ th SLA; $\beta_{0}, \beta_{1}, \beta_{2}$, and $\beta_{3}$ together with $\alpha_{1}$ and $\alpha_{2}$ are the model coefficients and intercepts (fixed effects). Here, $\Delta_{i k}(t)$ denotes the repeated measurement effects associated with the repeated observations of the same survey unit over the time. The estimated values of the model coefficients are presented in Table 3.

If there is a significant linear relationship between the each covariate in the model and the response variable, the associated coefficient will not equal zero. The hypothesis test to determine whether there is a significant linear relationship between $j$ th covariate (with the associated coefficient $b_{j}$ ) and the response variable is conducted as follows:

$$
\begin{aligned}
& H_{0}: b_{j}=0 \\
& H_{a}: b_{j} \neq 0
\end{aligned}
$$

The null hypothesis states that the coefficient is equal to zero, and the alter- 
Table 3: Initial Model Coefficient Estimates $\left(r^{2}=0.704\right)$

\begin{tabular}{||c|c|c||c|c|c||}
\hline \hline Parameter & Estimate & P-Value & Parameter & Estimate & P-Value \\
\hline \hline$\beta_{0}$ & -12.5072 & $<2.2 \mathrm{e}-16$ & $\alpha_{1}^{(7)}$ & 0.27 & 0.012126 \\
\hline$\beta_{1}$ & 15.1641 & $1.161 \mathrm{e}-05$ & $\alpha_{2}^{(1)}$ & 2.15 & 0.063241 \\
\hline$\beta_{2}$ & 0.0247 & 0.0004167 & $\alpha_{2}^{(2)}$ & 3.82 & 0.034875 \\
\hline$\beta_{3}$ & -1.0952 & $<2.2 \mathrm{e}-16$ & $\alpha_{2}^{(3)}$ & 1.03 & 0.040172 \\
\hline$\alpha_{1}^{(1)}$ & 4.43 & 0.001784 & $\alpha_{2}^{(4)}$ & 4.10 & 0.084561 \\
\hline$\alpha_{1}^{(2)}$ & 0.69 & 0.041948 & $\alpha_{2}^{(5)}$ & 0.07 & 0.018546 \\
\hline$\alpha_{1}^{(3)}$ & -0.28 & 0.049385 & $\alpha_{2}^{(6)}$ & 0.36 & 0.024651 \\
\hline$\alpha_{1}^{(4)}$ & -3.31 & 0.063432 & $\alpha_{2}^{(7)}$ & 0.20 & 0.102762 \\
\hline$\alpha_{1}^{(5)}$ & -0.06 & 0.154985 & $\alpha_{2}^{(8)}$ & 0.09 & 0.033245 \\
\hline$\alpha_{1}^{(6)}$ & 3.94 & 0.361382 & $\alpha_{2}^{(9)}$ & 0.42 & 0.010044 \\
\hline \hline
\end{tabular}

native hypothesis states that the slope is not equal to zero. The p-values are calculated for all model coefficient in Table 3.

In fitting GLMMs, difficulties associated with the correct variable selection are usually challenging and exert considerable influence on the outcomes. However, the first choice for model variables may not be the best choice. Several approaches to rectify the issue and re-select the model variables are discussed in the literature (e.g. Sauerbrei et al. (2006)). Here, we fit a step-wise (backward elimination method) mixed model, by which the insignificant parameters are to be excluded from the model gradually until the most significant model is determined. To do so, we combine the effect the households $H H T_{i k}^{(3)}$ and $H H T_{i k}^{(4)}$ to come up with the new model coefficient $H_{H} T_{i k}^{(3) *}$ with the associated covariate $\alpha_{1}^{(3) *}$. We also combine $H H T_{i k}^{(5)}$ and $H H T_{i k}^{(6)}$ to come up with the new coefficient $H H T_{i k}^{(5) *}$ with the associated covariate $\alpha_{1}^{(5) *}$. The effects of job related movements and movement with look for job reasons are also combined with the associated covariate $\alpha_{2}^{(4) *}$. The parameter estimates for the new model are presented in Table 4 . 
Table 4: Model coefficient estimates using the backward elimination method $\left(r^{2}=0.781\right)$

\begin{tabular}{||c|c|c||c|c|c||}
\hline \hline Parameter & Estimate & P-Value & Parameter & Estimate & P-Value \\
\hline \hline$\beta_{0}$ & -12.302 & $<0.0001$ & $\alpha_{1}^{(7)}$ & 0.31 & 0.0193 \\
\hline$\beta_{1}$ & 14.0091 & $<0.0001$ & $\alpha_{2}^{(1)}$ & 3.49 & 0.0304 \\
\hline$\beta_{2}$ & 0.02956 & $<0.0001$ & $\alpha_{2}^{(2)}$ & 4.01 & 0.0209 \\
\hline$\beta_{3}$ & -1.18261 & $<0.0001$ & $\alpha_{2}^{(3)}$ & 2.32 & 0.0021 \\
\hline$\alpha_{1}^{(1)}$ & 4.31 & 0.0015 & $\alpha_{2}^{(4) *}$ & 6.33 & 0.0095 \\
\hline$\alpha_{1}^{(2)}$ & 0.83 & 0.0390 & $\alpha_{2}^{(5)}$ & 1.01 & $<0.0001$ \\
\hline$\alpha_{1}^{(3) *}$ & -2.06 & 0.01790 & $\alpha_{2}^{(6)}$ & 0.97 & 0.0003 \\
\hline$\alpha_{1}^{(5) *}$ & -1.98 & 0.0240 & $\alpha_{2}^{(8)}$ & 0.15 & 0.0412 \\
\hline$\alpha_{1}^{(6)}$ & 3.80 & 0.0012 & $\alpha_{2}^{(9)}$ & 1.03 & 0.0143 \\
\hline
\end{tabular}

As presented in Table 4, all model parameter estimates are significant and the model accuracy measurement has improved when the backward elimination method is used. Model parameter estimates show that the existing difference between the supply and demand in the number of bedrooms has a positive effect on residential mobility. Similarly, the household income has a positive effect on the residential mobility. On the other hand, the tenure has a negative effect, meaning that generally, the longer Australian people live in a place the less less likely they are to move. According to the modelling results, couple families with children and lone parents with children aged over 15 are relocating more than other types of households.

The model residual diagnostics are plotted in Figure 4. These plots show the validity of the residual-leverage distributional assumption for the data used in modelling. Fitted values with large residuals and/or high leverage estimates may distort the accuracy of the fitted model and prediction as a consequence (?). Therefore, a fitted model with evenly distributed residuals and small leverage with less variability is expected to be appropriate for generalisation. In Figure 4, the left residuals-leverage plot with repeated measurements presents less variability rather than the the GLMM without repeated measurements (the right-side plot). This provides an evidence that the model with repeated mea- 
surements for this data set can capture more variability among the residuals associated with the model random effects.

Figure 4: Influence Residual plots for GLMM with the repeated measurements (on the left) and without the repeated measurements (on the right)
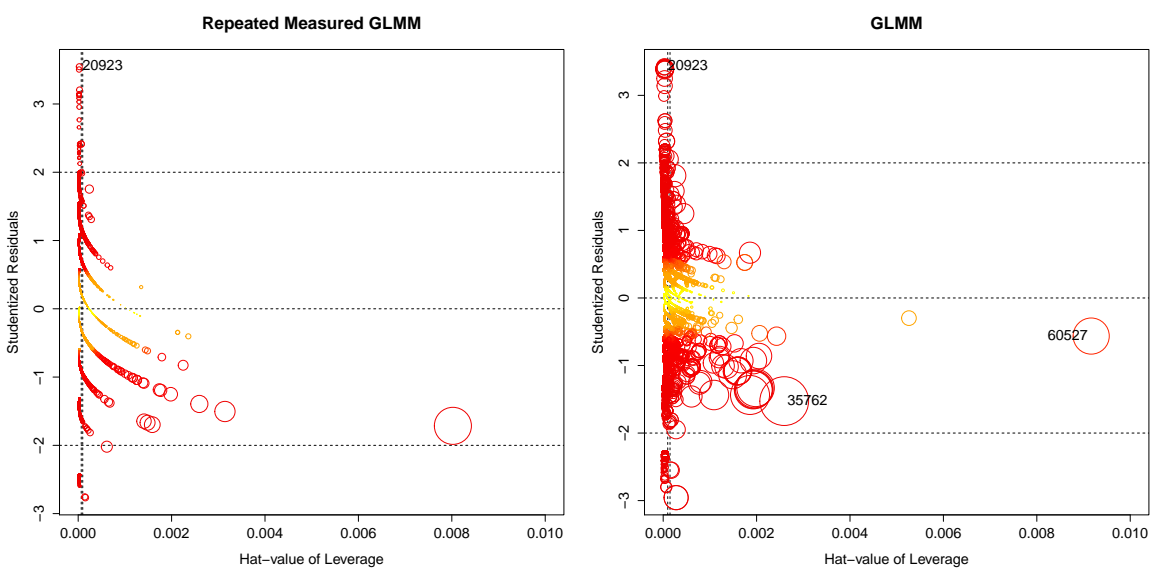

If the $i$ th households in HILDA within the $k$ th SLA relocated within a year, then, $\pi_{i k}^{(R)}=\operatorname{Pr}\left(I\left(y_{i k}^{(R)}\right)=1\right)=1$, otherwise $\pi_{i k}^{(R)}=0$. To validate the results obtained from the models presented in this paper the model predictions are to be compared with the HILDA data. Figure 4 illustrates the model-based prediction for residential mobility for those who have moved within a year based on the HILDA data and for those who have not moved. In each case the results of the models with and without the repeated measurements are presented to show how the repeated measurements will increase the model accuracy in estimates in our case study. It will be noted that the model-based predictions are presented in way to be ascending for the probability values. Based on the results presented in Figure 5, the model predictions are more accurate when adding the repeated measurements. 
Figure 5: Model-based predictions vs actual data
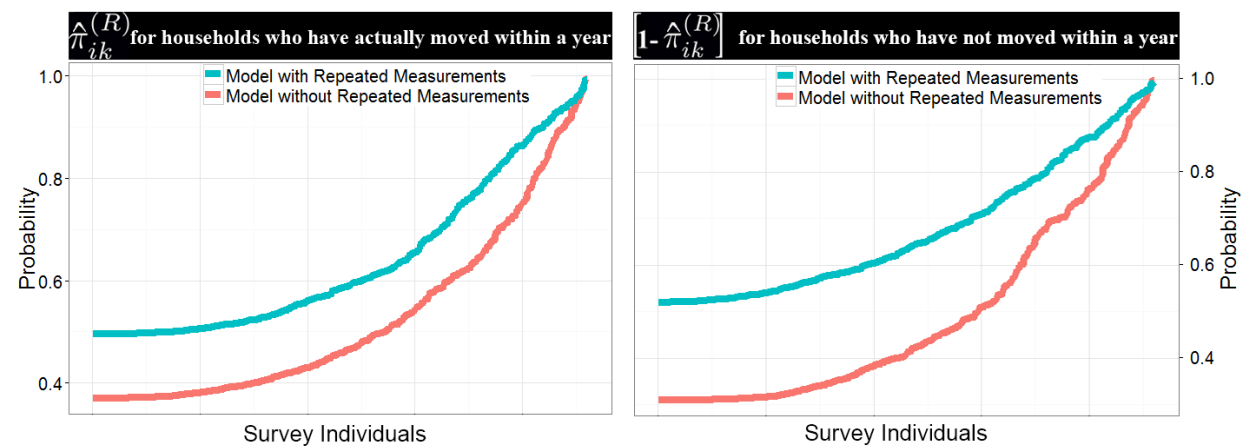

\section{Discussion}

Robust models of residential relocation have the power to incorporate the most important factors that influence a geographical area's popular perception and value of available services. Residential mobility is a process that has traditionally been modelled using aggregate forecasting that often provides a singular predicted state based on a certain statistical model and related assumptions. This study developed a logit mixed-effect model of trigger for a residential move at the household level while the repeated measurement effects are considered. The main attributes of this model are change in household income, household configuration, and the tenure of the household. HILDA survey data for 20012011 is used to estimate the coefficients in the logit model. This model, validated against existing datasets, provides some indication that choice modelling is proven to be an appropriate means of modelling the autonomous nature of relocation decision made by households. Not only does this model consider the area effects but also considers the effects of time and repeated measurements. Given the basis of this model, it is possible to calculate the probability of movements for non-sampled households in other parts of Australia.

Residential relocation predictions can be added to base demographic profiles and behaviours in simulating-based synthetic populations (Namazi-Rad et al. 
(2014b); Namazi-Rad et al. (2014a) ) to develop an agent-based simulation model that can re-create in silico the observed complex patterns emerging from social responses to changes in public policies or infrastructure configurations. How these choices affect the overall urban landscape is a product of a number of other interactions part of a larger research effort. However, the validity in the findings presented in this paper provides some guidance as to what predictive modelling tools can be integrated with other tools to provide the deeper understanding required for effective policy design.

\section{Acknowledgement}

This paper uses unit record data from the Household, Income and Labour Dynamics in Australia (HILDA) Survey. The HILDA Project was initiated and is funded by the Australian Government Department of Social Services (DSS) and is managed by the Melbourne Institute of Applied Economic and Social Research (Melbourne Institute). The findings and views reported in this paper, however, are those of the author and should not be attributed to either DSS or the Melbourne Institute.

\section{References}

ABS, 2004. Census of Population and Housing: Socio-Economic Indexes for Area's (SEIFA): Australia 2001, Technical Paper, ABS catalogue no. 2039.0.55.001. Australian Bureau of Statistics- Commonwealth of Australia, Canberra, Australia.

ABS, 2008. Socio-Economic Indexes for Areas (SEIFA) Technical Paper 2006, ABS catalogue no. 2039.0.55.001. Australian Bureau of Statistics- Commonwealth of Australia, Canberra, Australia.

Ahmed, S., Fallahpour, S., 2012. Shrinkage estimation strategy in quasilikelihood models. Statistics and Probability Letters 82, 2170-2179. 
Anderson, C., Verkuilen, J., Johnson, T., 2013. Applied Generalized Linear Mixed Models: Continuous and Discrete Data-For the Social and Behavioral Sciences. Springer.

Benedetti, A., Platt, R., Atherton, J., 2014. Generalized linear mixed models for binary data: Are matching results from penalized quasi-likelihood and numerical integration less biased? PLoS ONE 9, e84601.

Bhat, C., Guo, J., 2004. A mixed spatially correlated logit model: Formulation and application to residential choice modelling. Transportation Research Part B: Methodological 38, 147-168.

Black, D., Kalb, G., Kostenko, W., 2009. Location Economics: Spatial Mobility and Social Exclusion, Final report. Melbourne Institute of Applied Economic and Social Research, Melbourne, Australia.

Chambers, R., Clark, R., 2012. An Introduction to Model-Based Survey Sampling with Applications. Oxford University Press.

Chiou, J., Ma, Y., Tsai, C., 2012. Functional random effect time-varying coefficient model for longitudinal data. Stat: ISIs Journal for the Rapid Dissemination of Statistics Research 1, 75-89.

Claassen, R., Hellerstein, D., Kim, S., 2013. Using mixed logit in land use models: Can expectation-maximization (em) algorithms facilitate estimation? American Journal of Agricultural Economics 95, 419-425.

Demidenko, E., 2013. Mixed Models: Theory and Applications with R. John Wiley \& Sons, Hoboken, New Jesrsey.

Diggle, P., Sousa, I., Chetwynd, A., 2008. Joint modelling of repeated measurements and time-to-event outcomes: The fourth armitage lecture. Statistics in Medicine 27, 2981-2998.

Gad, A., Kholy, R.E., 2012. Generalized linear mixed models for longitudinal data. International Journal of Probability and Statistics 1, 67-73. 
Haynes, M., Western, M., Yu, L., Spallek, M., 2005. Methods for categorical longitudinal survey data: Understanding employment status of australian women, in: The Household, Income and Labour Dynamics in Australia (HILDA) Survey, HILDA Survey Research Conference, University of Melbourne, VIC, (1-24). 29/09/05 - 30/09/05.

Hedeker, D., Gibbons, R., 2006. Longitudinal Data Analysis. John Wiley \& Sons, Hoboken, New Jersey.

Henderson, C., 1975. Best linear unbiased estimation and prediction under a selection model. Biometrics 31, 423-447.

Hu, W., Cox, L., Wright, J., Harris, T., 2008. Understanding firms relocation and expansion decisions using self-reported factor importance rating. The Review of Regional Studies 38, 67-88.

Ibeas, ., Cordera, R., dellOlio, L., Coppola, P., 2013. Modelling the spatial interactions between workplace and residential location. Transportation Research Part A 49, 110-122.

Jelleyman, T., Spencer, N., 2008. Residential mobility in childhood and health outcomes: A systematic review. Journal of Epidemiology and Community Health $62,584-592$.

Jun, M., 2013. The effects of housing preference for an apartment on residential location choice in seoul: A random bidding land use simulation approach. Land Use Policy 43, 395-405.

Kan, K., 1999. Expected and unexpected residential mobility. Journal of Urban Economics 45, 72-96.

Lee, B., Waddell, P., 2010. Residential mobility and location choice: A nested logit model with sampling of alternatives. Transportation 37, 587-601.

Louviere, J., Meyer, R., 2008. Formal choice models of informal choices: What choice modeling research can (and can't) learn from behavioral theory, in: 
Malhotra, N. (Ed.), Review of Marketing Research Volume 4. ME Sharpe Inc, New York, USA.

Marschak, J., 1960. Binary choice constraints on random utility indications, in: Stanford Symposium on Mathematical Methods in the Social Sciences, Stanford, CA. pp. 312-329.

Mccullagh, P., 1983. Quasi-likelihood functions. Annals of Statistics 11, 59-67.

Minnucc, C., 2008. Community Profile 2007. Hobart City Council.

Namazi-Rad, M., Huynh, N., Barthelemy, J., P, P.P., 2014a. Synthetic population initialization and evolution- agent-based modelling of population aging and household transitions, in: Dam, H., Pitt, J., Xu, Y., Governatori, G., Ito, T. (Eds.), PRIMA 2014: Principles and Practice of Multi-Agent Systems - Lecture Notes in Computer Science- Volume 8861. Springer.

Namazi-Rad, M., Mokhtarian, P., Perez, P., 2014b. Generating a dynamic synthetic population - using an age-structured two-sex model for household dynamics. PLoS ONE 9, e94761.

Namazi-Rad, M., Steel, D., 2015. What level of statistical model should we use in small area estimation? Australian and New Zealand Journal of Statistics $57,275-298$,

NorthernTerritoryGovernment, 2007. Darwin city waterfront. Archived from the original on 4 June 2007. Retrieved 13 May 2007. .

Oishi, S., Schimmack, U., 2010. Residential mobility, well-being, and mortality. Journal of Personality and Social Psychology 98, 980-994.

OSullivan, A., 2009. Urban Economics. McGraw-Hill.

Pagliara, F., Wilson, A., 2010. The state-of-the-art in building residential location models, in: Pagliara, F., Preston, J., Simmonds, D. (Eds.), Residential Location Choice: Models and Applications, Springer, Berlin. pp. 1-20. 
Sauerbrei, W., Meier-Hirmer, C., Benner, A., Royston, P., 2006. Multivariable regression model building by using fractional polynomials: Description of sas, stata and r programs. Computational Statistics \& Data Analysis 50, 34643485 .

Sergeant, J., Ekerdt, D., Chapin, R., 2008. Measurement of late-life residential relocation: Why are rates for such a manifest event so varied? Journal of Gerontology: SOCIAL SCIENCES 63B, S92S98.

South, S., Crowder, K., 1997. Residential mobility between cities and suburbs: Race, suburbanization, and back-to-the-city moves. Demography 34, 525-538.

Thurstone, L., 1927. A law of comparative judgment. Psychological Review 34, $273-286$.

Train, K., 2009. Discrete Choice Methods with Simulation. Cambridge University Press.

Vega, A., Reynolds-Feighan, A., 2009. A methodological framework for the study of residential location and travel-to-work mode choice under central and suburban employment destination patterns. Transportation Research Part A 43, 401-419.

Wall, W., Williams, H., 1970. Longitudinal Studies and the Social Sciences. Heinemann, London.

Watson, N., Wooden, M., 2014. Re-engaging with survey non-respondents: Evidence from three household panels. Journal of the Royal Statistical Society: Series A (Statistics in Society) 177, 499-522.

Wedderburn, R., 1974. Quasi-likelihood functions, generalized linear models, and the gaussnewton method. Biometrika 61, 439-447.

Zeger, S., Liang, K., 1986. Longitudinal data analysis for discrete and continuous outcomes. Biometrics 42, 121-130. 


\section{Appendix}

The definition of general linear mixed models (LMM) is: (Demidenko (2013); Namazi-Rad and Steel (2015))

$$
\mathbf{Y}=\mathbf{X} \beta+\mathbf{Z u}+\mathbf{e}
$$

where $\mathbf{Z}$ is an $N \times K$ matrix of random-effect regressors. Here, $\mathbf{u}$ and $\mathbf{e}$ are assumed to be distributed independently with mean zero and covariance matrices $\mathbf{G}$ and $\mathbf{R}$, respectively. Note that, $\mathbf{G}$ and $\mathbf{R}$ depend on the variance components $\theta=\left[\theta_{1}, \ldots, \theta_{m}\right]^{\prime}$.

$$
\Sigma=\operatorname{Var}\left(\begin{array}{l}
\mathbf{u} \\
\mathbf{e}
\end{array}\right)=\left(\begin{array}{cc}
\mathbf{G} & \mathbf{0} \\
\mathbf{0} & \mathbf{R}
\end{array}\right), \quad E(\mathbf{e})=\mathbf{0} \quad \& \quad E(\mathbf{u})=\mathbf{0}
$$

The mean vector and covariance matrix for $\mathbf{Y}$ are respectively, $\mu_{Y}=\mathbf{X} \beta$ and $\mathbf{V}=\mathbf{Z G} \mathbf{Z}^{\prime}+\mathbf{R}$. (?)

The best linear unbiased estimation (BLUE) of the fixed effects $\beta$ and best linear unbiased prediction (BLUP) of the random effects $\mathbf{u}$ in LMM are:

$$
\begin{gathered}
\mathbf{X}^{\prime} \mathbf{R}^{-1} \mathbf{X} \tilde{\beta}+\mathbf{X}^{\prime} \mathbf{R}^{-1} \mathbf{Z} \tilde{\mathbf{u}}=\mathbf{X}^{\prime} \mathbf{R}^{-1} \mathbf{Y} \\
\mathbf{Z}^{\prime} \mathbf{R}^{-1} \mathbf{X} \tilde{\beta}+\left(\mathbf{Z}^{\prime} \mathbf{R}^{-1} \mathbf{Z}+\mathbf{G}^{-1}\right) \tilde{\mathbf{u}}=\mathbf{Z}^{\prime} \mathbf{R}^{-1} \mathbf{Y}
\end{gathered}
$$

Note that, within the statistical literature, it is conventional to use 'estimation' for fixed effects and "predictions" for random effects. Considering the equations in $(16), \mathbf{V}^{-1}$ can be defined in order to simplify the calculations.

$$
\mathbf{V}^{-1}=\mathbf{R}^{-1}-\mathbf{R}^{-1} \mathbf{Z}\left(\mathbf{Z}^{\prime} \mathbf{R}^{-1} \mathbf{Z}+\mathbf{G}^{-1}\right)^{-1} \mathbf{Z}^{\prime} \mathbf{R}^{-1}
$$

Then, we have:

$$
\mathbf{G} \mathbf{Z}^{\prime} \mathbf{V}^{-1}=\left(\mathbf{Z}^{\prime} \mathbf{R}^{-1} \mathbf{Z}+\mathbf{G}^{-1}\right)^{-1} \mathbf{Z}^{\prime} \mathbf{R}^{-1}
$$

The plug-in formulas for $\tilde{\beta}$ and $\tilde{u}$ can be found as a result of solving the equations above.

$$
\begin{aligned}
& \tilde{\beta}=\left(\mathbf{X}^{\prime} \mathbf{V}^{-1} \mathbf{X}\right)^{-1} \mathbf{X}^{\prime} \mathbf{V}^{-1} \mathbf{Y} \\
& \tilde{\mathbf{u}}=\mathbf{G} \mathbf{Z}^{\prime} \mathbf{V}^{-1}\left(\mathbf{Y}-\mathbf{X}^{\prime} \tilde{\beta}\right) .
\end{aligned}
$$


The maximum likelihood estimator for the parameter $\beta$ is the same as BLUE for this model parameter.

An ordinary linear model analysis assumes independence between any two observations. However, two appealing design features, repeated measures and cluster sampling, both create correlations among some observations and therefore require a more general model. In longitudinal data (repeated measures over time), two observations from the same subject will typically be correlated by sharing the same characteristics and therefore not independent. The central difference between a LMM with repeated measures and the ordinary LMM is adding a new factor to the model to account for the correlated error in the dependent variables. In order to consider such inter-dependencies, the variance matrix $\Sigma$ should be partitioned differently, as follows:

$$
\Sigma_{\Delta_{k}(t)}=\left(\begin{array}{cc}
\mathbf{G} & \mathbf{W}_{\Delta(t)}, \\
\mathbf{W}_{\Delta(t)}^{\prime} & \mathbf{R}
\end{array}\right)
$$

denotes the correlated variance-covariance structure of area-specific effects with repeated measurements over the time

In the case where $\mathrm{Z}$ is a $N \times K$ dimensional matrix that includes 1 s and 0 s which assigns the same value of $\mathbf{u}$ to all the rows referring to the units within the $k$ th area, the $\mathbf{V}=\mathbf{W}_{\Delta(t)}^{\prime} \mathbf{G} \mathbf{W}_{\Delta(t)}+\mathbf{R}$. (Diggle et al. (2008)) 